respiratory tract infections were observed in patients treated with DZP vs PBO; the majority were mild. We observed four thromboembolic events: one in the $24 \mathrm{mg} / \mathrm{kg}$ DZP group and three in the PBO group.

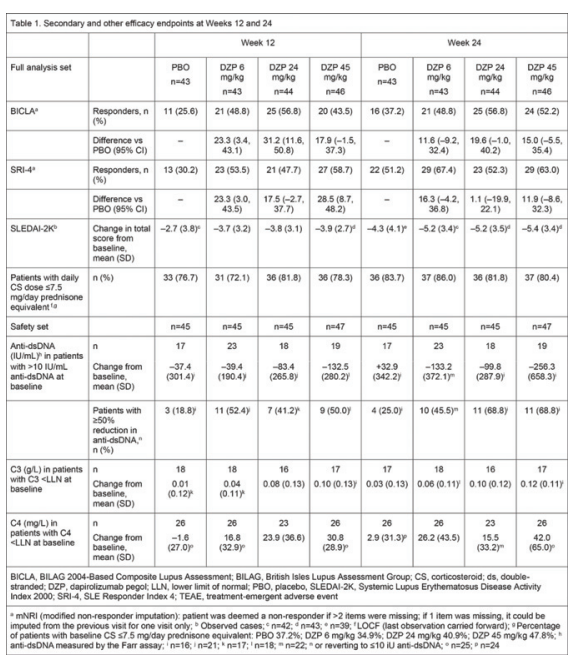

\begin{tabular}{|c|c|c|c|c|}
\hline & $\begin{array}{l}\text { PBO } \\
n=45\end{array}$ & $\begin{array}{l}\mathrm{DZP} 6 \mathrm{mg} / \mathrm{kg} \\
\quad \mathrm{n}=45\end{array}$ & $\begin{array}{c}\mathrm{DZP} 24 \\
\mathrm{mg} / \mathrm{kg} \\
\mathrm{n}=45\end{array}$ & $\begin{array}{l}\mathrm{DZP} 45 \\
\mathrm{mg} / \mathrm{kg} \\
\mathrm{n}=47\end{array}$ \\
\hline Serious TEAEs, $n$ (\%) & $5(11.1)$ & $1(2.2)$ & $4(8.9)$ & $3(6.4)$ \\
\hline TEAEs of interest, $n(\%)$ & $9(20.0)$ & $8(17.8)$ & $11(24.4)$ & $9(19.1)$ \\
\hline $\begin{array}{l}\text { Thromboembolic } \\
\text { events }\end{array}$ & $3(6.7)$ & 0 & $1(2.2)$ & 0 \\
\hline $\begin{array}{l}\text { Moderate to } \\
\text { severe infections }\end{array}$ & $6(13.3)$ & $8(17.8)^{\mathrm{a}}$ & $11(24.4)$ & $8(17.0)$ \\
\hline \multicolumn{5}{|c|}{$\begin{array}{l}\text { DZP, dapirolizumab pegol; PBO, placebo, TEAE, treatment-emergent adverse event } \\
\text { a One TEAE was of mild intensity but was deemed to be a TEAE of interest by the } \\
\text { investigator for the interim analysis }\end{array}$} \\
\hline
\end{tabular}

Conclusion: DZP appeared to be well tolerated as TEAEs were generally balanced across the treatment groups. Numerically greater improvements relative to PBO were observed consistently across multiple efficacy endpoints and biomarkers; however, for the primary endpoint no pre-specified dose-response relationship model fit the observed BICLA response rates at Week 24 with statistical significance. The potential for deriving clinical benefit from DZP in patients with SLE warrants further investigation.

Acknowledgement: Supported by UCB Pharma and Biogen.

Disclosure of Interests: Richard Furie Grant/research support from: Biogen, UCB Pharma, but not in the last 12 months, Consultant for: Biogen, UCB Pharma, but not in the last 12 months, lan N. Bruce Grant/research support from: Genzyme Sanofi, GlaxoSmithKline, Consultant for: AstraZeneca, Eli Lilly, GlaxoSmithKline, ILTOO Pharma, Medlmmune, Merck Serono, Speakers bureau: GlaxoSmithKline, UCB Pharma, Thomas Dörner Grant/research support from: Eli Lilly, Janssen, Roche, UCB Pharma, Consultant for: Eli Lilly, Janssen, Roche, UCB Pharma, Speakers bureau: Eli Lilly, Janssen, Manuel Gustavo Leon: None declared, Piotr Leszczynski Grant/research support from: AbbVie, Amgen, Bristol-Myers Squibb, Immupharma, Janssen Cilag, Novartis, Roche, UCB Pharma, Consultant for: AbbVie, Amgen, Berlin Chemie, Eli Lilly, Novartis, Roche, Sandoz, UCB Pharma, Speakers bureau: AbbVie, Amgen, Berlin Chemie, Eli Lilly, Novartis, Roche, Sandoz, UCB Pharma, Murray B Urowitz Grant/research support from: GSK, Consultant for: BMS, Celgene, GSK, Lilly, UCB, Birgit Haier Shareholder of: UCB Pharma, Employee of: UCB Pharma, Teri Jimenez Employee of: UCB Pharma, Catherine Barbey Shareholder of: Biogen, Employee of: Biogen, Jiajun Liu Employee of: Biogen, Christian Stach Shareholder of: UCB Pharma, Employee of: UCB Pharma DOI: 10.1136/annrheumdis-2019-eular.5529

\section{FRI0196 \\ TREATMENT OF SYSTEMIC LUPUS ERYTHEMATOSUS PATIENTS WITH THE IMMUNOPROTEASOME INHIBITOR KZR-616: RESULTS FROM THE FIRST 2 COHORTS OF AN OPEN-LABEL PHASE 1B DOSE ESCALATION TRIAL}

Richard Furie ${ }^{1}$, Darrin Bomba ${ }^{2}$, Maria Dall'era ${ }^{3}$, Massiel Prieto ${ }^{4}$, Janet Anderl ${ }^{2}$, Jinhai Wang ${ }^{2}$, Christopher Kirk', Niti Goel ${ }^{2,5} .{ }^{1}$ Northwell Health, Great Neck, NY, United States of America; ${ }^{2}$ Kezar Life Sciences, South San Francisco, CA, United States of America; ${ }^{3}$ University of California San Francisco, San Francisco, CA, United States of America; ${ }^{4}$ Hope Clinical Trials, Miami, FL, United States of America; ${ }^{5}$ Duke University School of Medicine, Durham, NC, United States of America

Background: Nonspecific proteasome inhibitors, e.g., bortezomib (BTZ), target both the constitutive and immuno- proteasomes and are standard of care for multiple myeloma. While BTZ has been used to treat refractory systemic lupus erythematosus (SLE) and lupus nephritis (LN), it is associated with adverse events (AEs) that limit its broad use. KZR-616, a first-in-class selective inhibitor of the immunoproteasome, is highly active and well tolerated in murine $\mathrm{SLE}^{1}$. In a Phase $(\mathrm{Ph}) 1$ healthy volunteer (HV) study, KZR-616 subcutaneously (SC) at 30 and $45 \mathrm{mg}$ weekly (QW) was shown to be safe, be well tolerated and achieve the target level of immunoproteasome inhibition ${ }^{2}$. We report here the preliminary safety and efficacy of KZR-616 in the Ph 1b portion of Study KZR616-002 in active SLE patients (pts) (NCT03393013).

Objectives: The $1^{\circ}$ objective of this first-in-patient study is to assess the safety and tolerability of KZR-616. The $2^{\circ}$ objectives are to evaluate pharmacokinetics (PK) and determine the KZR-616 doses for the Ph 2 portion of the study. Pharmacodynamics (PD) and efficacy are also being assessed.

\begin{tabular}{|l|l|c|c|}
\hline $\begin{array}{l}\text { Assessments } \\
\text { Mean (SD) }\end{array}$ & Timepoint & $\begin{array}{c}\text { Cohort 1 } \\
\mathbf{n = 6}\end{array}$ & $\begin{array}{c}\text { Cohort 2 } \\
\mathbf{n}=3\end{array}$ \\
\hline \multirow{3}{*}{ SLEDAI } & BL & $9.7(1.97)$ & $10.0(2.00)$ \\
\cline { 2 - 4 } & W13 & $7.2(3.35)$ & $6.0(2.83)$ \\
\hline \multirow{3}{*}{ CLASI } & BL & $5.5(2.07)$ & $7.3(1.16)$ \\
\cline { 2 - 4 } TJC & W13 & $3.6(1.95)$ & $4.5(2.12)$ \\
\hline \multirow{3}{*}{ SJC } & BL & $14.3(8.14)$ & $11.7(4.93)$ \\
\cline { 2 - 4 } & W13 & $7.6(6.99)$ & $8.5(3.54)$ \\
\hline \multirow{3}{*}{ PhGA } & BL & $11.0(6.03)$ & $9.3(3.06)$ \\
\cline { 2 - 4 } & W13 & $4.4(6.07)$ & $3.5(4.95)$ \\
\hline \multirow{3}{*}{ PtGA } & BL & $58.3(19.24)$ & $63.3(26.50)$ \\
\cline { 2 - 4 } & W13 & $39.8(24.67)$ & $40.0(14.14)$ \\
\cline { 2 - 4 } & BL & $57.5(25.79)$ & $73.3(11.59)$ \\
\hline & W13 & $43.6(31.64)$ & $35.0(7.07)$ \\
\hline
\end{tabular}

Methods: This open-label multicenter dose escalation trial enrolled SLE pts (per Systemic Lupus International Collaborating Clinics Classification Criteria) with SLE Disease Activity Index (SLEDAI) $\geq 4$ despite stable background immunosuppressant, antimalarial, and/or corticosteroid ( $\leq 20 \mathrm{mg}$ prednisone equivalent) therapy. Patients received KZR-616 at $45 \mathrm{mg}$ (Cohort 1) or $60 \mathrm{mg}$ (Cohort 2) SC QW through Week 13 (W13) with 12 weeks of follow up. Cohort $2 a$ is currently enrolling pts using intrapatient dose escalation from 30 to $60 \mathrm{mg}$. Safety data include AEs, vitals, electrocardiograms and laboratory tests. Efficacy measures include the SLEDAI, Cutaneous Lupus Erythematosus Disease Area and Severity Index (CLASI), 28 tender (T) and swollen (S) joint counts (JC), Physician Global Assessment (PhGA), and Patient Global Assessment (PtGA) in evaluable pts (receive $\geq 1$ month of KZR-616; non-evaluable pts can be replaced).

Results: We enrolled 13 pts: 8 in Cohort 1, 5 in Cohort 2. The pts were $100 \%$ female; Baseline $(B L)$ median SLEDAl was 10.0. All pts received at least 1 dose of KZR-616. In each cohort, 3 pts withdrew due to withdrawal of consent prior to $W 13$. Overall, the $45 \mathrm{mg}$ dose was well tolerated with no SAEs; all AEs were mild in intensity. The most common AEs were injection site erythema (62.5\%), nausea (25\%), and injection site pruritus (25\%).Cohort $2(60 \mathrm{mg})$ enrollment was halted, as all pts experienced vomiting within 8-24 hours (h) of their first dose, which typically resolved within $24 \mathrm{~h}$. One pt had an SAE of thrombotic microangiopathy. Two pts permanently reduced their dose to $45 \mathrm{mg}$. After 2 doses at $45 \mathrm{mg}$, another pt successfully re-escalated to $60 \mathrm{mg}$. In 
Cohort $2 a(n=5$ to date), there has been 1 SAE of localized herpes zoster. In pts, PK and PD were similar to that achieved for the same doses in $\mathrm{HV}^{1}$. Efficacy data for evaluable pts are shown in the table. Two (33\%) and $2(67 \%)$ evaluable pts in Cohorts 1 and 2, respectively, had a SLEDAI improvement of $\geq 4$ points from BL at W13.

Conclusion: KZR-616 dosed at $45 \mathrm{mg} \mathrm{SC} \mathrm{QW} \mathrm{appears} \mathrm{to} \mathrm{be} \mathrm{safe} \mathrm{and}$ well tolerated and showed evidence of disease suppression at W13 in active SLE pts on stable background therapy. The $\mathrm{Ph} 2$ doses in the first randomized placebo-controlled trial with KZR-616 in active LN pts on mycophenolate and prednisone will be 30 and $45 \mathrm{mg}$. Efforts are ongoing to evaluate $\mathrm{KZR}-616$ doses $\geq 60 \mathrm{mg}$ using step-up dosing.

\section{REFERENCES :}

[1] Muchamuel, et al. ARD 2018. 77:A685.

[2] Lickliter, et al. ARD 2018. 77:A1413.

Disclosure of Interests: Richard Furie Grant/research support from: Biogen, UCB Pharma, but not in the last 12 months, Consultant for: Biogen, UCB Pharma, but not in the last 12 months, Darrin Bomba Shareholder of: Stockholder in Kezar Life Sciences, Employee of: Employee of Kezar Life Sciences, Maria Dall'Era Grant/research support from: University has received funds to serve as a site on this clinical study, Consultant for: On Data Monitoring Committee for Janssen, Biogen, and Genentech; on Steering Committee for EMD Serono., Massiel Prieto Grant/research support from: Site has received funds to conduct this clinical trial, Janet Anderl Shareholder of: Stockholder in Kezar Life Sciences, Employee of: Employee of Kezar Life Sciences, Jinhai Wang Shareholder of: Shareholder in Kezar Life Sciences, Employee of: Employee of Kezar Life Sciences, Christopher Kirk Shareholder of: Shareholder and option holder in Kezar Life Sciences, Consultant for: Consultant for Amgen, Employee of: Employee of Kezar Life Sciences, Niti Goel Shareholder of: Own stock options in Kezar Life Sciences., Employee of: Corporate officer of Kezar Life Sciences.

DOI: 10.1136/annrheumdis-2019-eular.1955

\section{FRI0197 EFFICACY DATA OF MC2-03 (CICLOSPORIN 0.03\% AND $0.06 \%$ EYEDROPS) IN SJOGREN'S PATIENTS WITH SEVERE KERATITIS: OUTCOMES OF THE NORTHERN LIGHTS PHASE 2B TRIAL}

Frederic Gomez ${ }^{1}$, Johan Selmer ${ }^{1}$, Morten Præstegaard ${ }^{1}$, Miguel Teus ${ }^{2}$, Luis Pablo ${ }^{3}$, Maite Sainz de la Maza ${ }^{4}$, Steffen Heegaard ${ }^{5} .{ }^{1}$ MC2 Therapeutics, Hørsholm, Denmark; ${ }^{2}$ University of Alcalá, Madrid, Spain; ${ }^{3}$ University Hospital Miguel Servet, Zaragoza, Spain; ${ }^{4}$ Institute Clinic of Ophthalmology, Hospital Clinic of Barcelona, Barcelona, Spain; ${ }^{5}$ Department of Ophthalmology/University Hospital Rigshospitalet, Copenhagen, Denmark

Background: Sjögren's syndrome is one of the most common rheumatic autoimmune disorders (1). It is a chronic systemic autoimmune disease in which immunomediated inflammation, characterized by lymphocytic infiltration of exocrine glands and epithelia, causes secretory gland dysfunction leading to dryness of the main mucosal surfaces (2) including dry eye with severe keratitis. Ciclosporin is a well-known immunosuppressant with anti-inflammatory properties through inhibition of cell-mediated reactions (inhibit the release of cytokines including IL-2).

Objectives: To report the clinical efficacy of MC2-03 (ciclosporin $0.03 \%$ and $0.06 \%$ eyedrops) in Sjogren's patients with severe keratitis from a 6 month clinical trial.

Methods: The NORTHERN LIGHTS trial is a randomized, double masked, controlled multicentre European trial that assessed MC2-03 $0.03 \%$, MC2$030.06 \%$, vehicle and best-standard-of-care (BSC) for the treatment of moderate-to-severe dry eye disease (patients having baseline corneal fluorescein staining (CFS) score 3 or 4 ). The full analysis set consisted of a total of 255 patients with 66 patients having a documented history of Sjögren's syndrome. The objective of the trial was to evaluate safety and efficacy of MC2-03 after treatment once daily for 6 months followed by a 3 month safety follow-up. The primary efficacy endpoint was the proportion of patients achieving 2-grade improvement in CFS. CFS was evaluated using the modified Oxford grading system: a 7-point ordinal scale $(0,0.5$, and 1 to 5$)$, where grade 0 represents complete corneal clearing (absence of staining dots).

Results: The 66 patients with documented history of Sjögren's syndrome constituted $25.9 \%$ of the trial population with $90.9 \%$ of the patients being females $(n=60)$. Among the Sjögren patients, $58 \%(n=38)$ had severe dry eye (CFS 4) at baseline. Data analyzed for observed cases showed that the proportion of Sjogren's patients achieving 2-grade improvement of CFS (baseline CFS 3 and CFS 4) was higher in MC2-03 groups com pared to the control groups (Table 1). When patients treated with any active were compared to patients treated with any control, a 2-grade improvement of the CFS was observed in $51.9 \%$ patients in the combined active group vs. $26.7 \%$ for the combined control group $(p=0.0620)$.

Table 1. Sjögren patients with 2-grade reduction in CFS

\begin{tabular}{|c|c|c|c|c|c|c|}
\hline MC2-03 0.06\% & MC2-03 0.03\% & vehicle & BSC & Combined active & Combined control & Significance \\
\hline $7 / 16(43.8 \%)$ & $7 / 11(63.6 \mathrm{~K})$ & $4 / 14(28.6 \%)$ & $4 / 16(25 \%)$ & $14 / 27(51.9 \%)$ & $8 / 30(26.7 \%)$ & $\mathrm{p}=0.0620$ \\
\hline
\end{tabular}

Using the same approach, the most severe patients, having CFS 4 at baseline, had a statistically significantly higher responder rate of $64.3 \%$ in the combined active group versus $21.1 \%$ in the combined control group $(p=0.0286)$.

Table 2. Severe Sjögren patients with 2-grade reduction in CFS

\begin{tabular}{|c|c|c|}
\hline Combined active & Combined control & Significance \\
\hline $9 / 14(64.3 \%)$ & $4 / 19(21.1 \%)$ & $p=0.0286$ \\
\hline
\end{tabular}

Conclusion: Data from the NORTHERN LIGHTS trial show that MC2-03 ciclosporin eyedrops has the potential to be a safe, well-tolerated and efficient treatment in Sjogren's patients.

\section{REFERENCES}

[1] Fox, et al. 2000

[2] Kassan, et al. 2004

Disclosure of Interests: Frederic Gomez Employee of: MC2 Therapeutics, Johan Selmer Employee of: MC2 Therapeutics, Morten Præstegaard Employee of: MC2 Therapeutics, Miguel Teus Grant/research support from: Alcon, Allergan, Novartis, Johnson \& Johnson, Consultant for: Alcon, Allergan, Novartis, Johnson \& Johnson, Santen, Speakers bureau: Alcon, Allergan, Johnson \& Johnson, Luis Pablo Consultant for: Alcon, Allergan, Santen, MSD, Speakers bureau: Alcon, Allergan, Santen, Thea MSD, Maite Sainz de la Maza Consultant for: Abbvie, Dompe, Santen, Speakers bureau: Bausch \& Lomb, Brill, Esteve, Santen, Thea, Steffen Heegaard Speakers bureau: Alcon, Santen, Thea

DOI: 10.1136/annrheumdis-2019-eular.4246

\begin{tabular}{|l|l}
\hline FRI0198 & ANTIMALARIAL AGENTS DIMINISH WHILE \\
METHOTREXATE, AZATHIOPRINE AND \\
MYCOPHENOLIC ACID INCREASE BAFF LEVELS IN \\
SYSTEMIC LUPUS ERYTHEMATOSUS
\end{tabular}

Borja Hernández-Breijo ${ }^{1}$, Alvaro Gomez ${ }^{2,3}$, Sofia Soukka ${ }^{2,3}$, Petter Johansson ${ }^{2,3}$ Ioannis Parodis ${ }^{2,3}$. 'La Paz University Hospital, Immuno-Rheumatology Research Group, IdiPaz, Madrid, Spain; ${ }^{2}$ Karolinska Institutet, Division of Rheumatology, Department of Medicine, Stockholm, Sweden; ${ }^{3}$ Karolinska University Hospital, Rheumatology, Stockholm, Sweden

Background: Elevated levels of the B cell activating cytokine BAFF (also known as BLyS) have been associated with active systemic lupus erythematosus (SLE), and the anti-BAFF monoclonal antibody belimumab has been approved as an add-on to standard-of-care SLE treatment, the latter mainly comprising glucocorticoids, antimalarial agents (AMA) and other immunosuppressive treatments.

Objectives: To investigate the effect of AMA and three other commonly used in SLE immunosuppressive agents (methotrexate, azathioprine, mycophenolate mofetil/sodium) on serum BAFF levels.

Methods: Access to data from the phase III clinical trials of belimumab BLISS-52 ( $n=865 ;$ NCT00424476) and BLISS-76 ( $n=819 ;$ NCT00410384) was granted by GlaxoSmithKline (Uxbridge, UK); a total of 1684 SLE patients were analysed. Serum samples obtained prior to belimumab initiation were stored at $-80^{\circ} \mathrm{C}$ until BAFF level determination by ELISA. The non-parametric Mann-Whitney $U$ test was used to compare BAFF level distributions between treatment groups. Linear regression models were applied to determine independence. 